\title{
Activation of Constitutive Androstane Receptor Ameliorates Renal Ischemia-Reperfusion-Induced Kidney and Liver Injury $\$$
}

\author{
You-Jin Choi, Dong Zhou, Anne Caroline S. Barbosa, Yongdong Niu, Xiudong Guan, \\ Meishu Xu, Songrong Ren, Thomas D. Nolin, Youhua Liu, and Wen Xie
}

Center for Pharmacogenetics and Department of Pharmaceutical Sciences (Y.-J.C., A.C.S.B., Y.N, M.X., S.R., W.X.), Department of Pathology, School of Medicine (D.Z., Y.L.), Center for Clinical Pharmaceutical Sciences, Department of Pharmacy and Therapeutics, School of Pharmacy (T.D.N), and Department of Pharmacology and Chemical Biology (W.X.), University of Pittsburgh, Pittsburgh, Pennsylvania; Department of Pharmacology, Shantou University Medical College, Shantou, Guangdong, China (Y.N.); and Department of Neurosurgery, Beijing Tiantan Hospital, Capital Medical University, Beijing, China (X.G.)

Received November 13, 2017; accepted January 5, 2018

\section{ABSTRACT}

Acute kidney injury (AKI) is associate with high mortality. Despite evidence of $\mathrm{AKI}$-induced distant organ injury, a relationship between AKI and liver injury has not been clearly established. The goal of this study is to investigate whether renal ischemiareperfusion (IR) can affect liver pathophysiology. We showed that renal IR in mice induced fatty liver and compromised liver function through the downregulation of constitutive androstane receptor (CAR; -90.4\%) and inhibition of hepatic very-low-density lipoprotein triglyceride (VLDL-TG) secretion (-28.4\%). Treatment of mice with the CAR agonist 1,4-bis[2-(3,5 dichloropyridyloxy)] benzene (TCPOBOP) prevented the development of AKIinduced fatty liver and liver injury, which was associated with the attenuation of AKI-induced inhibition of VLDL-TG secretion. The hepatoprotective effect of TCPOBOP was abolished in
$\mathrm{CAR}^{-1-}$ mice. Interestingly, alleviation of fatty liver by TCPOBOP also improved the kidney function, whereas CAR ablation sensitized mice to AKI-induced kidney injury and lethality. The serum concentrations of interleukin-6 (IL-6) were elevated by 27-fold after renal IR, but were normalized in TCPOBOP-treated AKI mice, suggesting that the increased release of IL-6 from the kidney may have mediated the AKI responsive liver injury. Taken together, our results revealed an interesting kidney-liver organ cross-talk in response to AKI. Given the importance of CAR in the pathogenesis of renal IR-induced fatty liver and impaired kidney function, fatty liver can be considered as an important risk factor for kidney injury, and a timely management of hepatic steatosis by CAR activation may help to restore kidney function in patients with AKI or kidney transplant.

\section{Introduction}

Acute kidney injury (AKI) is one of the serious complications of critical illness that is associated with significant mortality (Rewa and Bagshaw, 2014). AKI is characterized by the sudden impairment of kidney function, as measured by serum creatinine and urine volume (Khwaja, 2012), resulting in the retention of waste products. Ischemia is a common cause of $\mathrm{AKI}$, which can occur in many clinical situations, such as renal transplantation, partial nephrectomy, heart surgery, and hypoperfusion associated with sepsis. Hallmarks of ischemic AKI include inflammation, disruption of the apical brush border of tubular cells, and death of the renal tubular epithelial cells (Devarajan, 2006; Wu et al., 2007). Previous studies have shown that apoptosis and necrosis mainly occur

The work was supported in part by the National Institutes of Health [Grants DK083952, ES023438]. W.X. was supported in part by the Joseph Koslow Endowed Professorship from the University of Pittsburgh School of Pharmacy. https://doi.org/10.1124/mol.117.111146.

S This article has supplemental material available at molpharm. aspetjournals.org. in tubular epithelial cells following ischemic AKI (Shi et al., 2000). Although ischemia-induced kidney injury has been well documented, the effects of renal ischemia on distant organs need further investigation.

Earlier studies have suggested organ cross-talk between the injured kidney and distant organs, such as the lungs, liver, heart, gut, and brain (Doi and Rabb, 2016). More recent data highlight the importance of immune response, activation of inflammatory cascades, and an alteration of transcriptional events in distant organs during the renal injury. Hepatic dysfunction has been reported in patients suffering from AKI (Chertow et al., 1995). Liver injury after the ischemic renal injury or nephrectomy is characterized by hepatocyte vacuolization, necrosis, and apoptosis with inflammatory changes (Park et al., 2011, 2012). Several studies have suggested mechanisms for the interaction between the injured kidney and liver. In one example, renal injury increased the intestinal expression of interleukin-17A (IL-17A) and caused small intestinal injury (Park et al., 2011). These events resulted in hepatic inflammation and increased the release of tumor

ABBREVIATIONS: AKI, acute kidney injury; ALT, alanine aminotransferase; apob100, apolipoprotein B100; AST, aspartate aminotransferase; BUN, blood urea nitrogen; CAR, constitutive androstane receptor; CKD, chronic kidney disease; IL, interleukin; IR, ischemia-reperfusion; MTTP, microsomal triglyceride transfer protein; PCR, polymerase chain reaction; TCPOBOP, 1,4-bis[2-(3,5 dichloropyridyloxy)] benzene; TUNEL, terminal transferase dUTP nick-end labeling; VLDL-TG, very-low-density lipoprotein triglyceride; WT, wild-type. 
necrosis factor $\alpha$ and IL- 6 from the liver into the circulation. Reactive oxygen species and oxidative stress are also important players in AKI-induced liver injury. Renal ischemiareperfusion (IR) reduces glutathione levels in the liver and blood through the inhibition of glutamate-cysteine ligase, the first rate-limiting enzyme of glutathione synthesis (Shang et al., 2016). Lipid peroxidation is also increased in the liver as evidenced by the elevated level of malondialdehyde. In contrast, administration of glutathione protects the liver from renal IR-induced injury, which was associated with decreased levels of malondialdehyde (Golab et al., 2009).

AKI can resolve naturally, but if not, it may progress to chronic kidney disease (CKD) (Venkatachalam et al., 2015). The relationship between $\mathrm{CKD}$ and fatty liver has been controversial. Obesity, insulin resistance, diabetes, and hypertension are shared risk factors in the development of both CKD and fatty liver (Targher and Byrne, 2017). Several cross-sectional and longitudinal studies suggest that fatty liver is associated with an increased prevalence of CKD (Musso et al., 2014). Moreover, the severity of fatty liver is associated with an increased risk of CKD in diabetic and nondiabetic patients. Meanwhile, CKD may contribute to the development of fatty liver and insulin resistance (Axelsson et al., 2011; Pelletier et al., 2013).

The constitutive androstane receptor (CAR) was initially characterized as a xenobiotic nuclear receptor regulating a mammal's responses to xenotoxicants (Honkakoski et al., 1998; Wei et al., 2000). Recently results, including those published from our laboratory, have implicated this receptor in the control of energy metabolism, including the inhibition of lipogenesis (Gao and Xie, 2012). Activation of CAR inhibited hepatic steatosis in both the high-fat diet and ob/ob obese models (Gao et al., 2009). Activation of CAR has also been shown to relieve hepatic injuries, such as those caused by cholestasis and hyperbilirubinemia, through increased metabolism and excretion of endogenous and exogenous toxins. The expression and activity of CAR can be suppressed by inflammation (Pascussi et al., 2000; Assenat et al., 2004). Knowing that acute inflammatory response is an integral component in the pathogenesis of $\mathrm{AKI}$, it is interesting to know whether and how CAR plays a role in the kidney-liver cross-talk in the context of AKI.

In this study, we uncovered a novel function of CAR in mediating the kidney-liver cross-talk in AKI. Renal IR downregulated the expression and activity of CAR and decreased the expression of microsomal triglyceride transfer protein (MTTP), which resulted in a decreased very-low-density lipoprotein triglyceride (VLDL-TG) secretion and lipid accumulation and liver injury. Pharmacological activation of CAR alleviated IR-induced fatty liver and liver injury, leading to improved renal function.

\section{Materials and Methods}

Animals, Animal Surgery, and Drug Treatments. Eightweek-old male C57BL/6 mice were purchased from Jackson Laboratory (Bar Harbor, ME). The creation of $\mathrm{CAR}^{-1-}$ mice has been described previously (Wei et al., 2000). Mice were maintained ad libitum in a temperature-controlled animal facility. To induce renal IR-induced AKI, both kidneys were clamped to block blood flow for 30 minutes. After ischemia, clamps were released to start reperfusion, and mice were sacrificed after 24 or 48 hours. For studying the effect of CAR activation, the mice were randomly assigned, and treatment groups were administered 1,4-bis[2-(3,5 dichloropyridyloxy)] benzene (TCPOBOP; $1 \mathrm{mg} / \mathrm{kg}$ body weight per day, i.p.) for 2 days before the renal IR surgery. Vehicle groups received the same volume of corn oil. All chemicals were ordered from Sigma-Aldrich (St. Louis, MO). The use of animals complied with the guidelines established by the Institutional Animal Care and Use Committee of the University of Pittsburgh.

Histologic Analysis. The kidney and liver tissues were fixed in $10 \%$ neutral buffered formalin for 24 hours and then dehydrated, embedded in paraffin, sectioned at $4 \mu \mathrm{m}$, and stained with H\&E for general histology. Immunostaining was performed on the paraffin sections. Slides were incubated overnight with the primary anti-CAR antibody (sc-13065) from Santa Cruz Biotechnology (Santa Cruz, CA) diluted to $1: 100$ at $4^{\circ} \mathrm{C}$ in humid chambers, and were counterstained with hematoxylin. To detect lipid deposits in the liver, liver tissues were embedded in Tissue-Tek OCT compound (Sakura Finetek, Torrance, CA), and cryosections $(8 \mu \mathrm{m})$ were prepared and stained with Oil-Red O (Sigma-Aldrich, St. Louis, MO). Terminal transferase dUTP nick-end labeling (TUNEL) staining was performed by using the In Situ Cell Death Detection Kit from Roche Molecular Biochemicals (Mannheim, Germany). In brief, paraffin sections were treated with the TUNEL enzyme solution and counterstained with DAPI (4',6-diamidino-2-phenylindole). The slides were immediately evaluated by fluorescence microscopy for positively stained necrotic nuclei.

Serum and Tissue Biochemistry. Serum was separated from blood by low-speed centrifugation $\left(1500 \mathrm{~g}, 10\right.$ minutes, $\left.4^{\circ} \mathrm{C}\right)$, transferred into aliquots, and stored at $-80^{\circ} \mathrm{C}$ until analysis. Serum creatinine and blood urea nitrogen (BUN) levels were evaluated with the QuantiChrom Creatinine Assay Kit and the QuantiChrom Urea Assay Kit (BioAssay System, Hayward, CA), respectively. The serum levels of alanine aminotransferase (ALT), aspartate aminotransferase (AST) (Stanbio Laboratory, Boerne, TX), and total bilirubin (SigmaAldrich) were measured using commercial assay kits. The concentrations of IL- 6 were measured by an enzyme-linked immunosorbent assay kit from R\&D Systems (Minneapolis, MN). To measure hepatic lipid contents, liver lipids were extracted from homogenate prepared from $100 \mathrm{mg}$ of tissue using chloroform/methanol mix $(2: 1, \mathrm{v} / \mathrm{v})$. Triglycerides and cholesterol levels in the liver lipid extracts and serum were determined using colorimetric assay kits from Stanbio Laboratory.

Western Blot Analysis. Liver and kidney tissue lysates were prepared by homogenization using lysis buffer (50 mM HEPES, $150 \mathrm{mM} \mathrm{NaCl}, 1 \%$ Triton X-100, $5 \mathrm{mM}$ EGTA, $50 \mathrm{mM}$ glycerophosphate, $20 \mathrm{mM} \mathrm{NaF}, 1 \mathrm{mM} \mathrm{Na}_{3} \mathrm{VO}_{4}$, and $2 \mathrm{mM}$ phenylmethylsulfonyl fluoride) and centrifuged at 12,000 rpm for 10 minutes. Supernatants were collected, electrophoresed on $8 \%-15 \%$ SDS-PAGE, and transferred onto polyvinylidene difluoride membrane (Millipore, Bedford, MA). Western blots were probed with the following antibodies: CAR (sc-13065), MTTP (sc-135994), and apolipoprotein B100 (sc-12332) from Santa Cruz; neutrophil gelatinase-associated lipocalin (MAB1857) from R\&D Systems; Caspase 3 (\#9662) from Cell Signaling Technology (Beverly, MA); and $\beta$-actin (A1978) from Sigma-Aldrich. Detection was performed by enhanced chemiluminescence using a Thermo ECL kit from Thermo Fisher (Waltham, MA).

RNA Isolation and Quantitative Real-Time Polymerase Chain Reaction. Total RNA was isolated using the TRIZOL reagent. RNase-free DNase I-treated total RNA was used to synthesize single-strand cDNA. A quantitative real-time polymerase chain reaction (PCR) was performed on an ABI 7300 Real-Time PCR System (Applied Biosystems, Foster City, CA). The primer sequences for the mouse Car are 5'-GGAGCGGCTGTGGAAATATTGCAT-3' and 5'-TCCATCTTGTAGCAAAGAGG CCCA-3'. The primer sequences for the mouse Cyp $2 b 10$ are 5'-GCATTTGTCTTGGTGAAAGCATT-3' and 5'-GGATGGACGTGAAGAAAAGGAA-3'. The primer sequences for the mouse $I l-6$ are $5^{\prime}$-CGACGGCCTTCCCTACTT-3' and 5'-TGGGAGTGGTATCCTCTGTGAA-3'. Melting curve analysis was performed to determine the specificity of amplification. Relative changes in gene expression were expressed as the fold change using 
$2^{-\Delta \Delta \mathrm{CT}}$. Gene expression was normalized to the expression of the control cyclophilin gene.

VLDL-TG Secretion Assay. After 4-hour fasting, mice were administered an intravenous injection of tyloxapol from SigmaAldrich at $500 \mathrm{mg} / \mathrm{kg}$ body weight. Blood samples were collected from the tail vein at $0,30,60$, and 120 minutes after the tyloxapol injection and plasma triglyceride levels were measured.

Transient Transfection and Luciferase Reporter Gene Assay. The pCMX-HA-mCAR and tk-PBRE constructs were described previously (Xie et al., 2000). HEK293T cells were transiently transfected with pCMX-HA-mCAR and tk-PBRE plasmids using Lipofectamine 2000 from Invitrogen (Carlsbad, CA). The pCMX$\beta$-gal plasmid was added as an internal control to monitor the transfection efficiency. After transfection, cells were incubated with IL-6 (50 U/ml) with dimethylsulfoxide or TCPOBOP $(200 \mathrm{nM})$ for 24 hours. The luciferase activity was normalized with the $\beta$-gal activity.
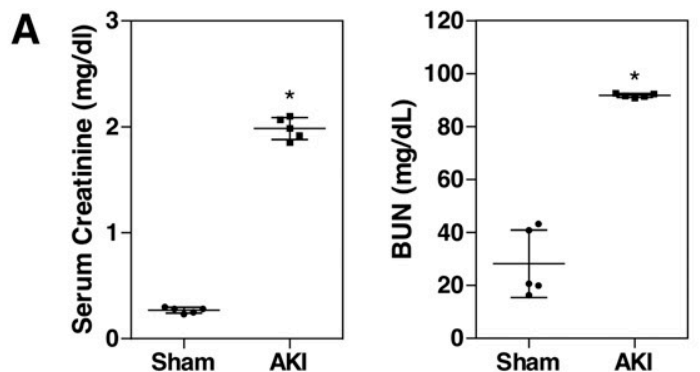

B
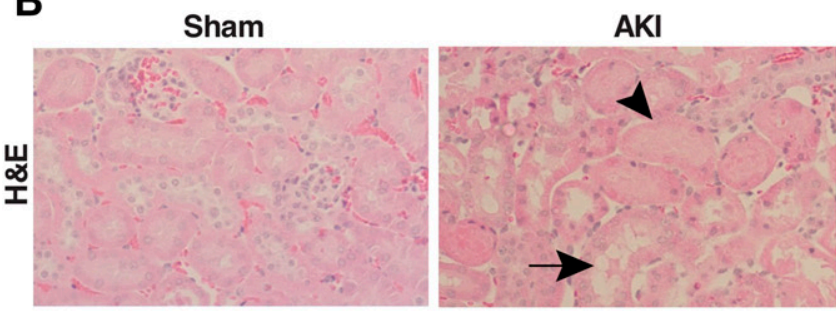

D
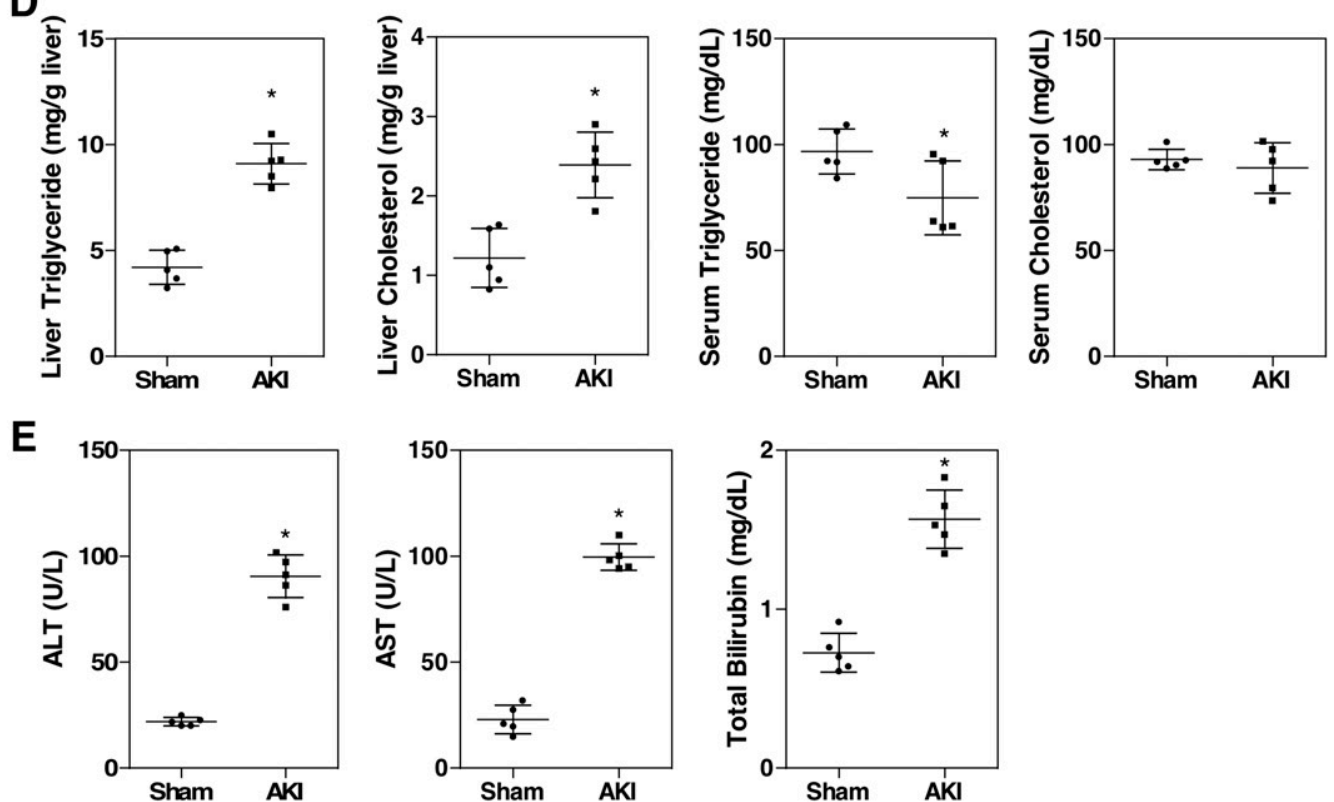

Fig. 1. Renal IR induces hepatic steatosis. (A) C57BL/6 male mice were subjected to sham surgery or 30 minutes of bilateral renal ischemia followed by 24 hours of reperfusion. Blood samples were collected to measure serum levels of creatinine and BUN. (B) Renal histology as shown by H\&E staining. Original magnification, $200 \times$. Arrow and arrowhead indicate tubular dilation and necrosis, respectively. (C) Macroscopic liver appearance (top) and liver histology as shown by H\&E staining (middle) and Oil-Red O staining (bottom). (D) Hepatic and serum lipid levels. (E) Serum ALT, AST, and total bilirubin levels. Results are presented as the mean \pm S.D. of five mice per group. $* P<0.05$, compared with the sham group. 
Compared with the normal renal structure in the sham group, the AKI group showed signs of severe renal pathologic changes, such as loss of tubular cell brush border, tubular dilatation, and necrosis (Fig. 1B). Next, we examined the liver, liver histology, and liver function. Unexpectedly, the livers of AKI mice were pale in color, and appeared fatty (Fig. 1C, top). When histology was examined by H\&E staining, cellular vacuolization was observed in the AKI mice (Fig. 1C, middle), which turned out to be lipid accumulation as confirmed by the Oil-Red $\mathrm{O}$ staining (Fig. 1C, bottom). Biochemical analysis of the lipids showed that the liver triglyceride and cholesterol levels were increased by 2 -fold in the AKI group, but the circulating levels of triglycerides and cholesterol were not significantly affected (Fig. 1D). In addition, the AKI mice developed acute hepatic dysfunction as evidenced by elevated plasma levels of ALT, AST, and total bilirubin (Fig. 1E).

Renal IR Decreases the Hepatic Expression of CAR. To understand the molecular mechanism by which renal IR induces hepatic steatosis, microarray was performed using a pooled liver sample from three mice in each group. The microarray data showed that the expression of nuclear receptor CAR was markedly decreased in the AKI group (data not shown), and this result was confirmed by quantitative real-time PCR (Fig. 2A), Western blotting (Fig. 2B), and immunohistochemistry (Fig. 2C). In addition to its xenobiotic functions, CAR is also known for its role in the inhibition of hepatic steatosis (Dong et al., 2009; Gao et al., 2009). These data led to our hypothesis that the downregulation of CAR may have played a role in the renal IR-induced fatty liver.

Treatment with the CAR Activator TCPOBOP Ameliorates Renal IR-Induced Fatty Liver. To determine whether CAR plays a role in renal IR-induced hepatic steatosis, mice were treated with the CAR activator TCPOBOP $(1 \mathrm{mg} / \mathrm{kg})$ for 2 days before being challenged with renal IR surgery. Whereas renal IR reduced the protein level of CAR in the liver, TCPOBOP treatment substantially restored the protein expression of CAR (Fig. 3A) and induced its nuclear translocation (Fig. 3B). Interestingly, the mRNA expression of CAR remained suppressed by renal IR upon the TCPOBOP treatment, regardless of whether TCPOBOP was administered before or after the IR surgery (Supplemental Fig. 1). The mechanism for the discrepancy between the protein and mRNA expression remains to be understood. As expected, the hepatic mRNA expression of Cyp2b10, a CAR target gene

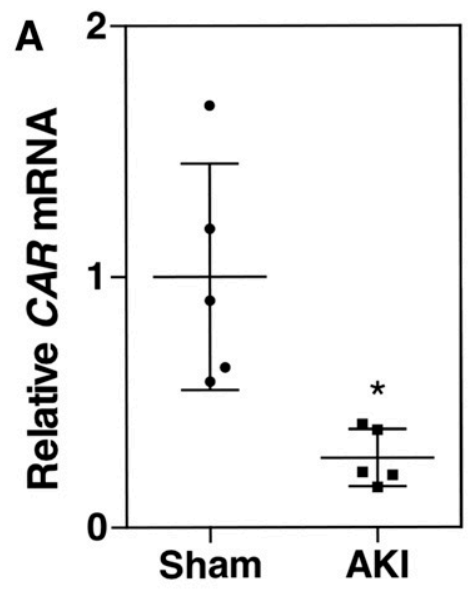

B

Sham
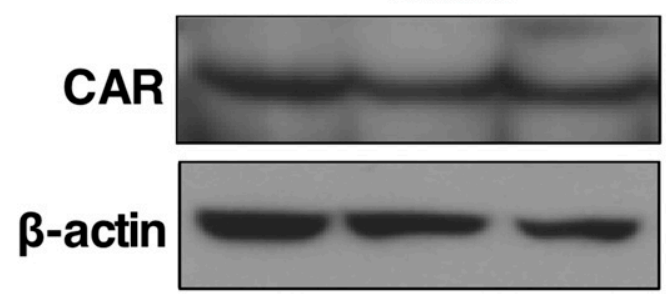

C

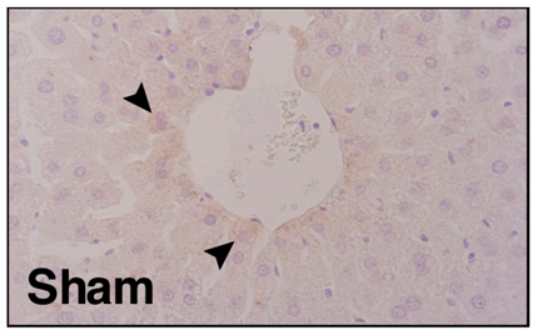

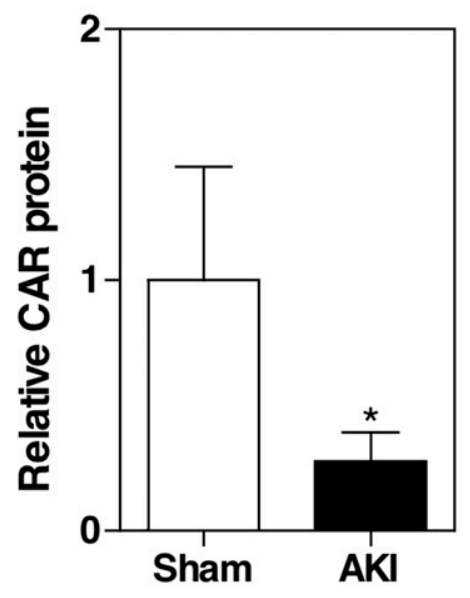

AKI

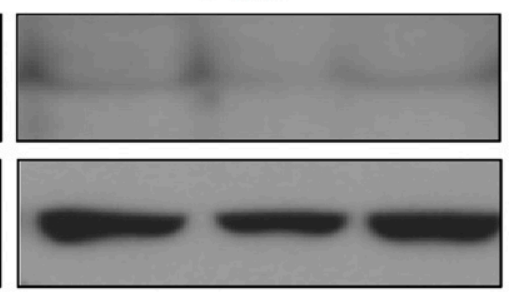

Fig. 2. Renal IR decreases the hepatic expression of CAR. Mice were the same as described in Fig. 1. (A) Liver $C A R$ mRNA expression was measured by quantitative real-time PCR. The relative gene levels are represented as fold change compared with the sham group. Results are presented as the mean \pm S.D. of five mice per group. $* P<0.05$, compared with the sham group. (B) Liver CAR protein expression was measured by Western blotting. Lanes represent individual mice. Shown on the top right is the densitometric quantification of the Western blotting results. (C) Liver CAR protein expression was detected by immunohistochemistry. Arrowheads indicate positive staining.

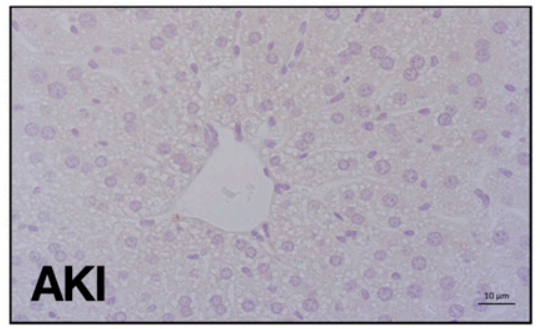


A
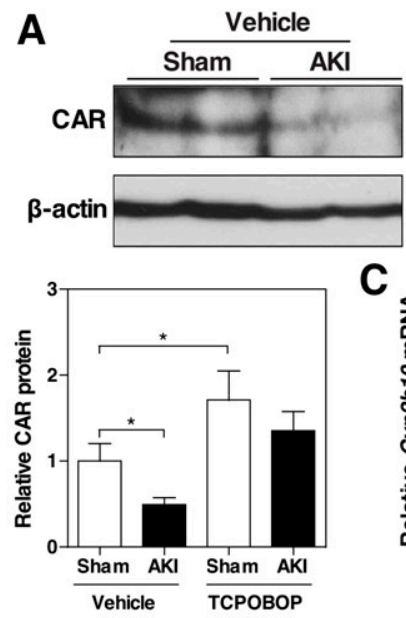

D
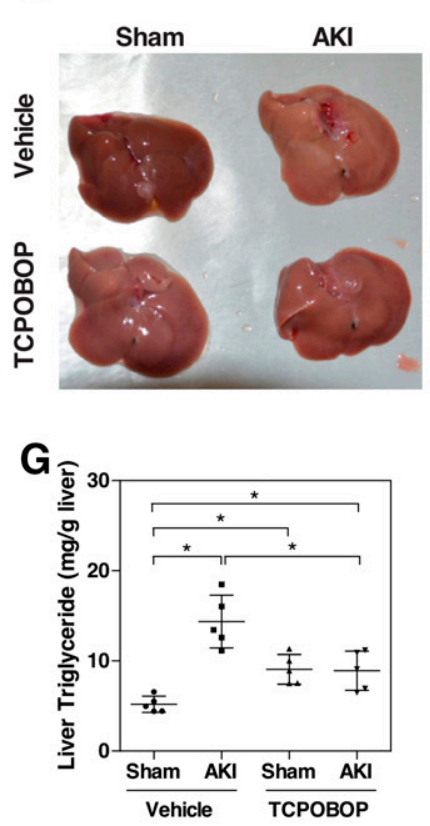

H

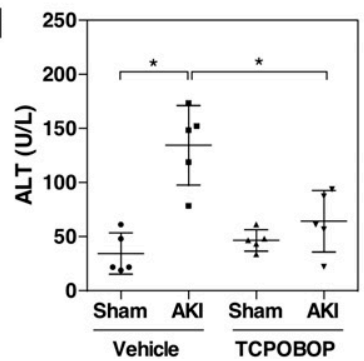

TCPOBOP
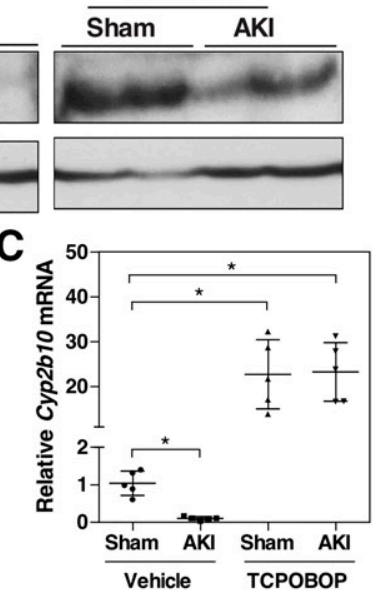

E
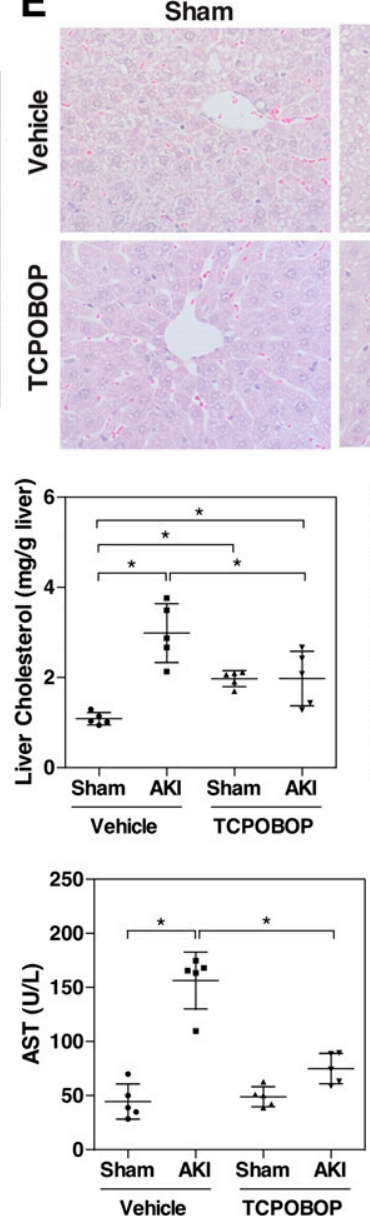

B

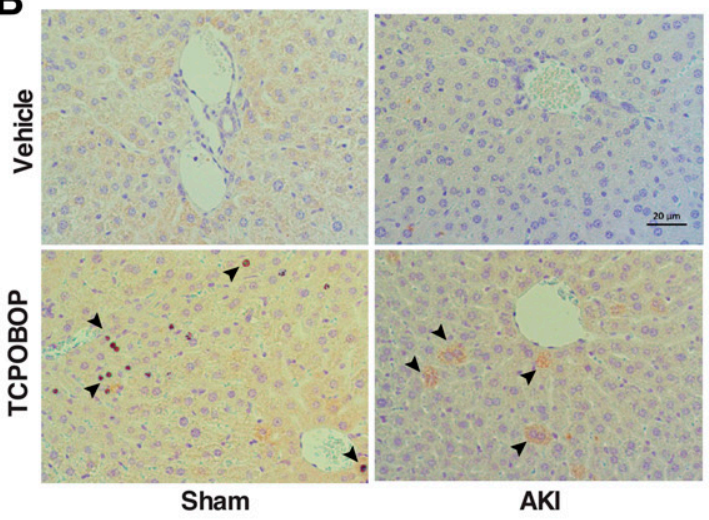

AKI

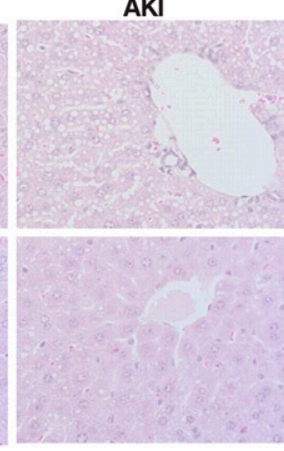

F

Sham

AKI
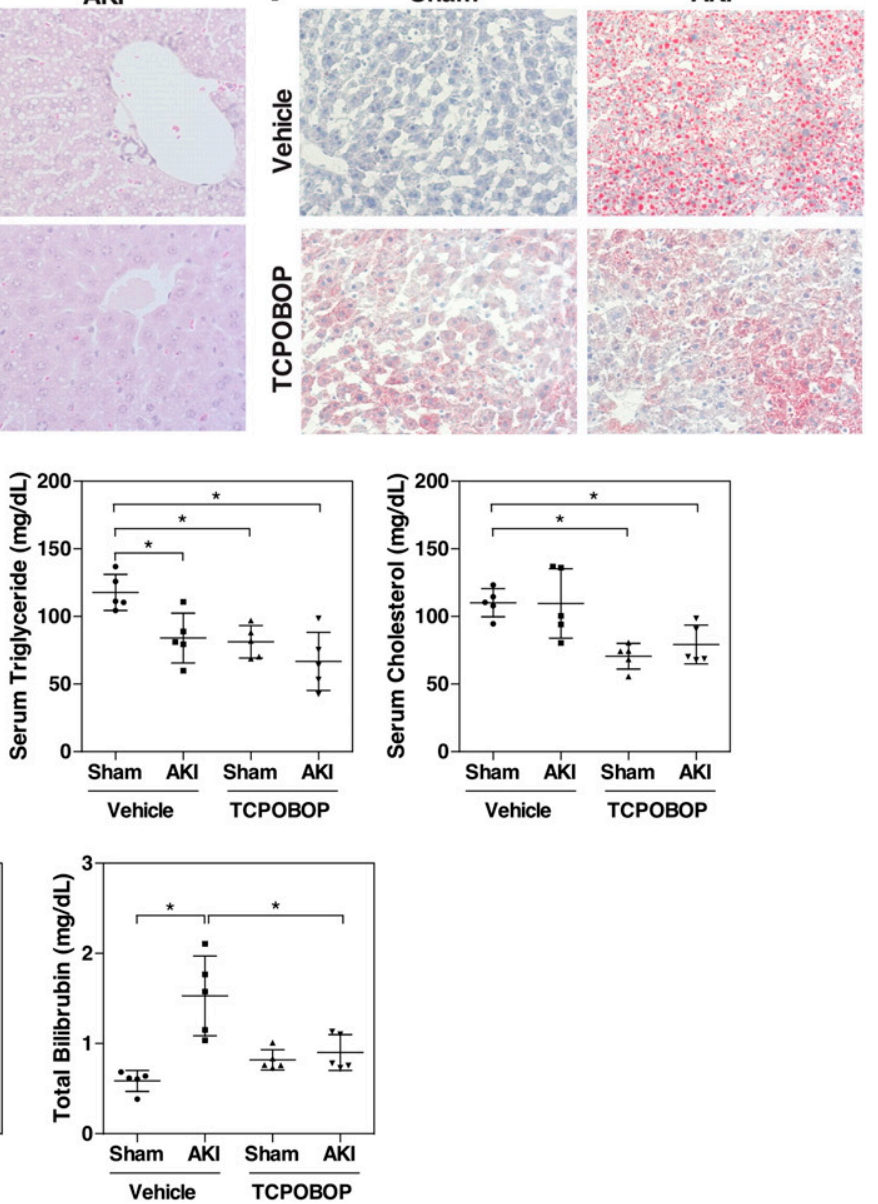

Fig. 3. Treatment with the CAR activator TCPOBOP ameliorates renal IR-induced fatty liver. C57BL/6 male mice were randomly assigned to four groups. Two treatment groups were administered TCPOBOP for 2 days ( $1 \mathrm{mg} / \mathrm{kg}$ body weight per day; i.p.) before receiving the sham surgery or renal IR 24 hours after the last doses of drugs. Two vehicle groups received the same volume of corn oil for 2 days before receiving the sham surgery or renal IR. (A) Liver CAR protein expression was measured by Western blotting. Shown on the bottom left is the densitometric quantification of the Western blotting results. (B) Liver CAR protein expression was detected by immunohistochemistry. Arrowheads indicate positive staining. (C) Cyp2b10 mRNA expression was measured by quantitative real-time PCR. The relative gene levels are represented as fold change compared with the vehicle-treated sham group. (D-F) Macroscopic liver appearance (D) and liver histology as shown by H\&E (E) and Oil-Red O staining (F). (G) Hepatic and serum lipid levels. (H) Serum ALT, AST, and total bilirubin levels. Results are presented as the mean \pm S.D. of five mice per group. $* P<0.05$, with the comparisons labeled.

that was suppressed by AKI, was induced/restored by TCPOBOP (Fig. 3C). The renal IR-induced steatosis was attenuated by TCPOBOP, as evidenced by the gross appearance (Fig. 3D), H\&E staining (Fig. 3E), Oil-Red $\mathrm{O}$ staining (Fig. 3F), and biochemical analysis of the hepatic and serum lipids (Fig. 3G). Of note, compared with the vehicle-treated sham mice, TCPOBOP-treated sham mice had a modest but significantly higher level of lipids (Fig. 3G), which might be secondary to the 
hepatomegaly effect of TCPOBOP or due to a decreased fatty acid oxidation, because the expression of $P P A R \alpha$ and $C p t 1 \alpha$ was reduced in the TCPOBOP-treated group (Supplemental Fig. 2), which was consistent with our previous report (Gao et al., 2009). In addition to relieving steatosis, treatment with TCPOBOP also improved liver function as evidenced by the normalized levels of AST, ALT, and total bilirubin (Fig. 3H).

Activation of CAR Blocks the Inhibitory Effects of Renal IR on VLDL-TG Secretion. To understand the molecular mechanisms underlying the renal IR-induced fatty liver, we investigated the effects of renal IR on hepatic gene expression with emphasis on genes associated with lipid metabolism. We found that the mRNA expression of the Mttp was significantly decreased in the AKI mice (data not shown), which was verified at the protein level by Western blotting (Fig. 4A). MTTP is necessary for the transfer of triglyceride and cholesteryl ester and the VLDL-TG secretion (Wetterau et al., 1997). It has been shown that downregulation of MTTP increases the susceptibility to hepatic steatosis due to decreased VLDL-TG secretion (Minehira et al., 2008). The serum level of apoB100, the main structural component of VLDL (Davidson and Shelness, 2000), was also decreased in the AKI mice (Fig. 4B). We then measured the VLDL-TG secretion in mice that had been injected with tyloxapol, a lipase inhibitor. The results showed that VLDL-TG secretion was substantially reduced in vehicle-treated AKI mice compared with vehicle-treated sham mice, but the inhibitory effect of AKI on VLDL-TG secretion was abolished by the treatment of TCPOBOP (Fig. 4C). Interestingly, in the sham groups, treatment with TCPOBOP resulted in a decrease in the basal VLDL-TG secretion compared with vehicle-treated sham mice, which indicated an inhibitory effect of CAR on VLDL-TG secretion (Fig. 4C). Consistent with the results of VLDL-TG secretion, treatment with TCPOBOP abolished the AKI responsive suppression of hepatic protein expression of Mttp (Fig. 4A) and the serum level of apoB100 (Fig. 4B).

The Attenuation of Renal IR-Induced Fatty Liver by TCPOBOP Is Abolished in $\mathbf{C A R}^{-1-}$ Mice. To assess whether the hepatoprotective effect of TCPOBOP is CAR- dependent, $\mathrm{CAR}^{-/-}$mice were treated with TCPOBOP for 2 days before receiving the renal IR surgery. Renal IR was effective in inducing fatty liver and liver injury in the $\mathrm{CAR}^{-1-}$ mice, as shown by the liver gross appearance (Fig. 5A), H\&E staining (Fig. 5B), Oil-Red O staining (Fig. 5C), and liver and serum lipid analysis (Fig. 5D). The renal IR-induced fatty liver in $\mathrm{CAR}^{-/-}$mice was not improved by the TCPOBOP treatment (Fig. 5, A-D). Treatment of $\mathrm{CAR}^{-1-}$ mice with TCPBOP had little effect in relieving the liver injury either, as evidenced by the measurements of AST, ALT, and total bilirubin levels (Fig. 5E). Renal IR also suppressed VLDL-TG secretion (Fig. 5F) and decreased the serum level of apoB100 (Fig. 5G) in the $\mathrm{CAR}^{-1-}$ mice, and these effects were not affected by the treatment of TCPOBOP.

Activation of CAR Attenuates Renal IR-Induced Kidney Injury. It has been reported that steatosis and the severity of steatosis in kidney transplant recipients were positively correlated with the serum creatinine levels (Glicklich et al., 1999; Mikolasevic et al., 2014), suggesting that steatosis has a negative effect on the function of transplanted kidneys. Knowing IR is an integral part of a kidney transplant and activation of CAR can attenuate renal IR responsive hepatic steatosis, we went on to test whether activation of CAR can protect the kidney from IR-induced injury. In this experiment, wild-type (WT) mice were treated with TCPOBOP for 2 days before receiving the renal IR surgery, and the mice were sacrificed and analyzed 48 hours later. Again, in this regimen, TCPOBOP treatment attenuated the renal IR responsive hepatic lipid accumulation, as confirmed by H\&E staining (Fig. 6A), Oil-Red O staining (Fig. 6B), and liver and serum lipid analysis (Fig. 6C). To our surprise, TCPOBOP-treated AKI mice also showed improvement of kidney function as indicated by their lower levels of serum creatinine and BUN compared with their vehicletreated counterparts (Fig. 6D). At the histologic level, TCPOBOP treatment reduced renal tubular damage and necrosis, as shown by H\&E (Fig. 6E). Tubular epithelial cell apoptosis was measured and quantified by TUNEL staining. As shown in Fig. $6 \mathrm{~F}$, apoptotic nuclei were abundant in vehicle-treated AKI
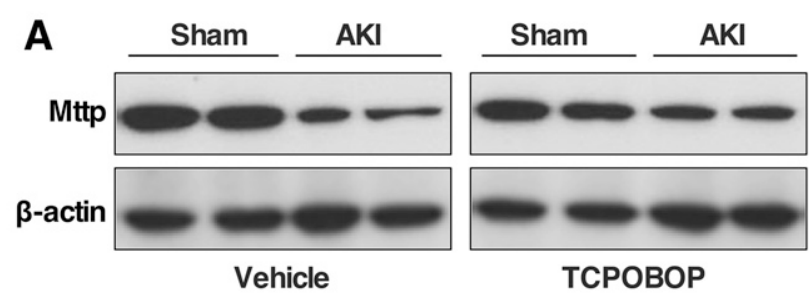

B

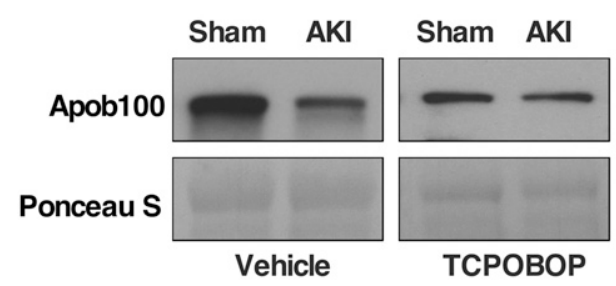

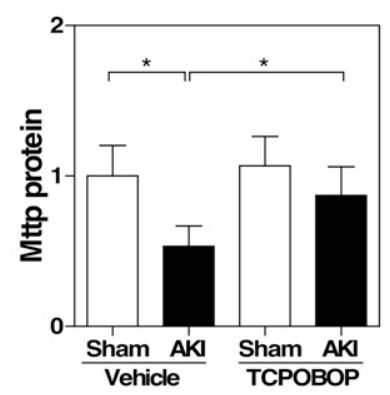

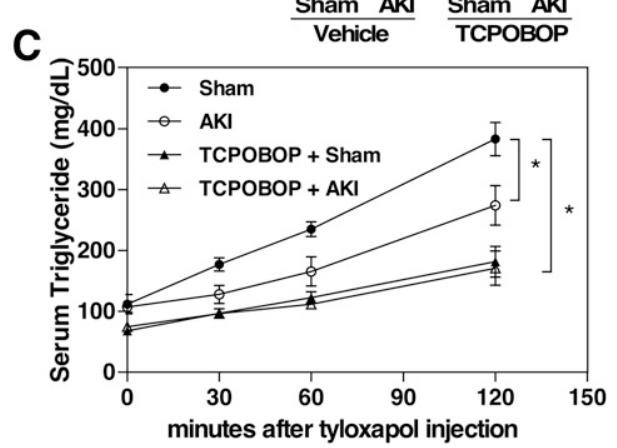

Fig. 4. Activation of CAR blocks the inhibitory effects of renal IR on VLDL-TG secretion. Mice were the same as described in Fig. 3. (A) Liver Mttp protein expression was measured by Western blotting. Shown on the right is the densitometric quantification of the Western blotting results. Each bar represents the mean \pm S.D. of five mice. (B) Serum apob100 level was detected by Western blotting. Ponceau S staining was used as a loading control. (C) VLDL-TG secretion assay. Mice were fasted for 4 hours before receiving an intravenous injection of tyloxapol (500 mg/kg body weight). Blood samples were collected at $0,30,60$, and 120 minutes and measured for serum concentrations of triglycerides. Each bar represents the mean \pm S.D. of three mice. ${ }^{*} P<$ 0.05 , with the comparisons labeled. 

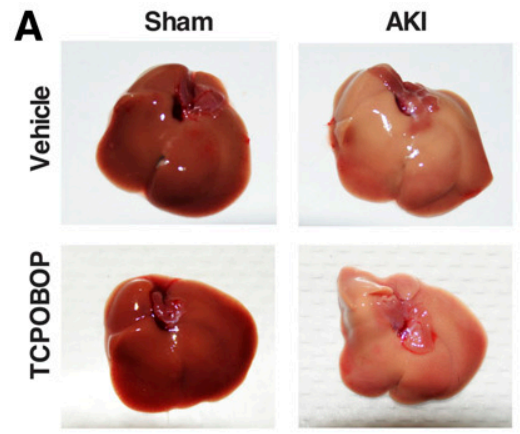
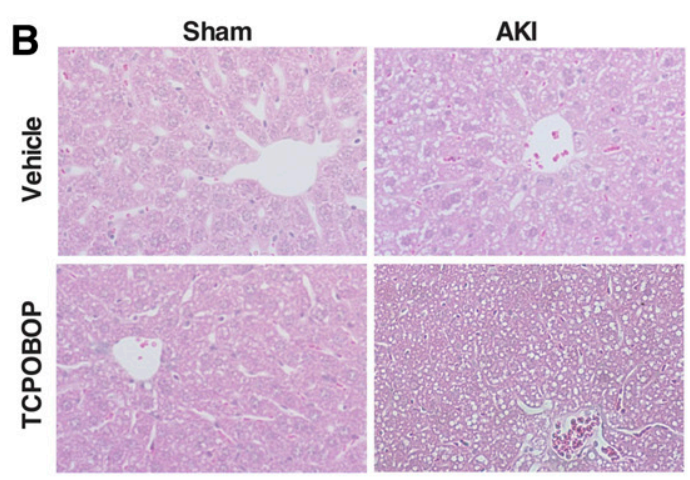

$\mathbf{F}$
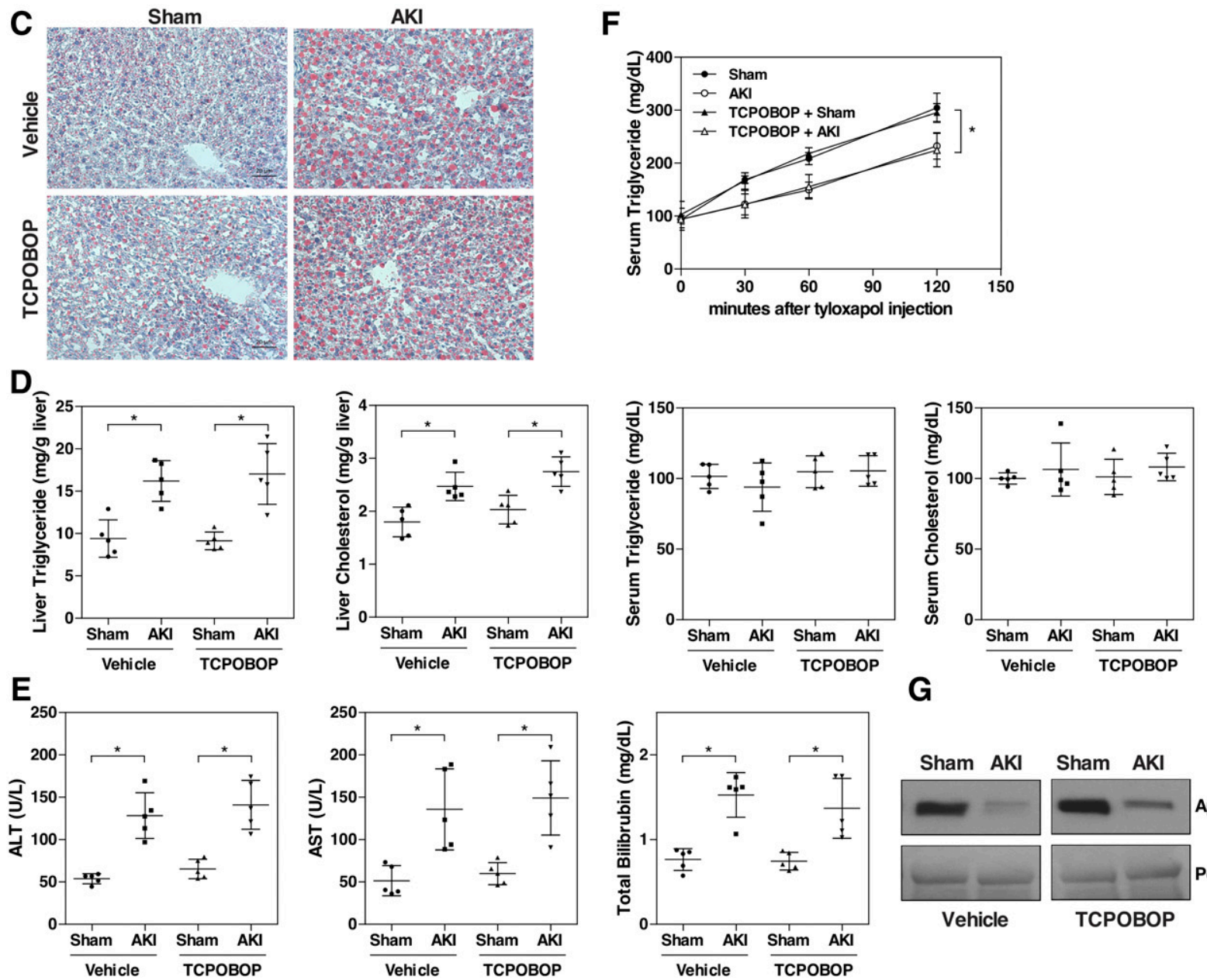

G

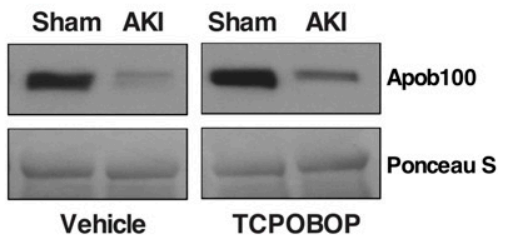

Fig. 5. The attenuation of renal IR-induced fatty liver by TCPOBOP is abolished in $\mathrm{CAR}^{-1-}$ mice. The experimental designs were the same described in Fig. 3 except that male $\mathrm{CAR}^{-1-}$ mice were used. (A-C) Macroscopic liver appearance (A) and liver histology as shown by H\&E (B) and Oil-Red O staining (C). (D) Hepatic and serum lipid levels. (E) Serum ALT, AST, and total bilirubin levels. (F) VLDL-TG secretion assay. (G) Serum apob100 was detected by Western blotting. Ponceau S staining was used as a loading control. Each bar represents the mean \pm S.D. of five mice. ${ }^{*} P<0.05$, with the comparisons labeled.

mice, whereas the TCPOBOP treatment significantly reduced the number of TUNEL-positive cells (Fig. 6F). Western blot analysis showed that renal IR responsive expression of neutrophil gelatinase-associated lipocalin, a biomarker of acute kidney injury (Shemin and Dworkin, 2011), was decreased in TCPOBOP-treated mice (Fig. 6G), as was the cleavage of caspase 3 (Fig. 6G). Compared with the liver, the expression of CAR was negligible in the kidney as shown by quantitative real-time PCR and Western blotting (Supplemental Fig. 3), consistent with a previous report (Choi et al., 1997).
CAR Ablation Sensitizes Mice to Renal IR-Induced Lethality. To determine whether CAR ablation affected an animal's sensitivity to renal IR-induced lethality, WT and $\mathrm{CAR}^{-1-}$ mice were subjected to the 30-minute ischemia/48-hour reperfusion model. Whereas all WT mice survived the renal IR, nearly $50 \%$ of the $\mathrm{CAR}^{-1-}$ mice died after 48 hours (Fig. 7A). Histologic analysis (Fig. 7B) and TUNEL (Fig. 7C) staining showed greater tubular injury in the surviving $\mathrm{CAR}^{-/-}$mice compared with their WT counterparts. 
A

A
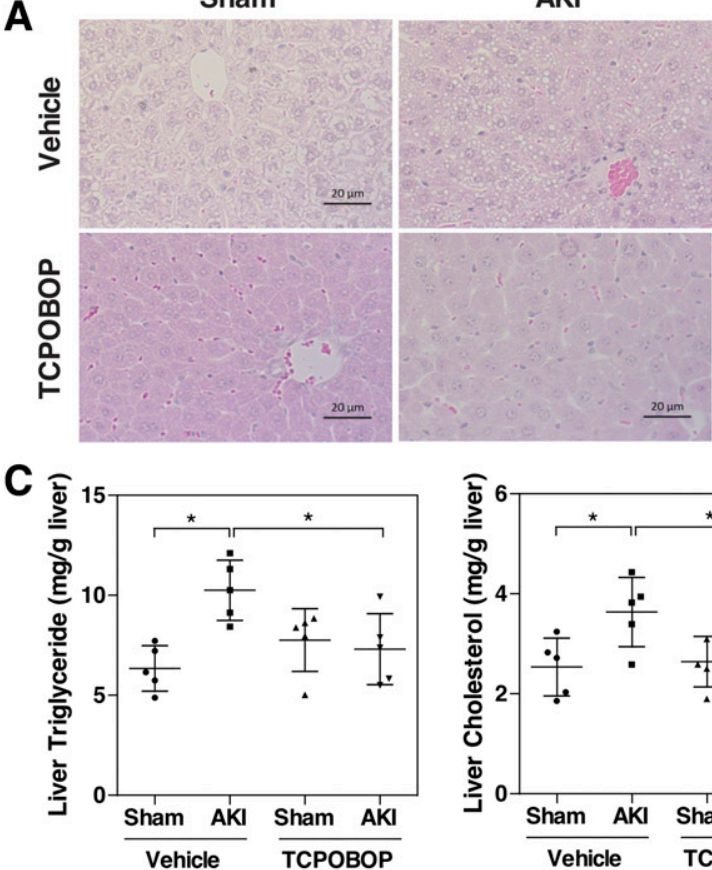

D

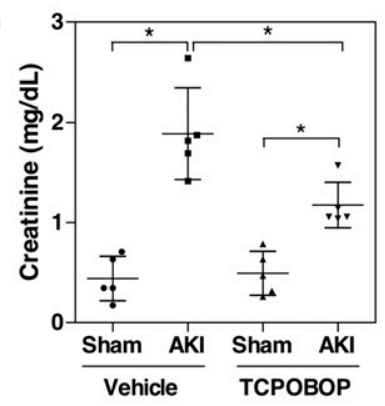

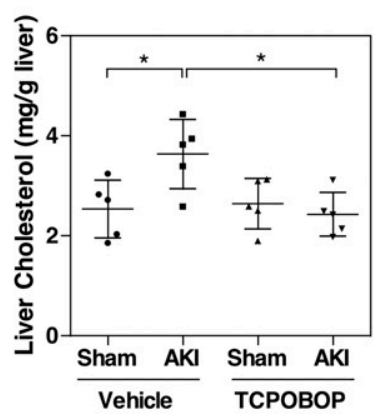
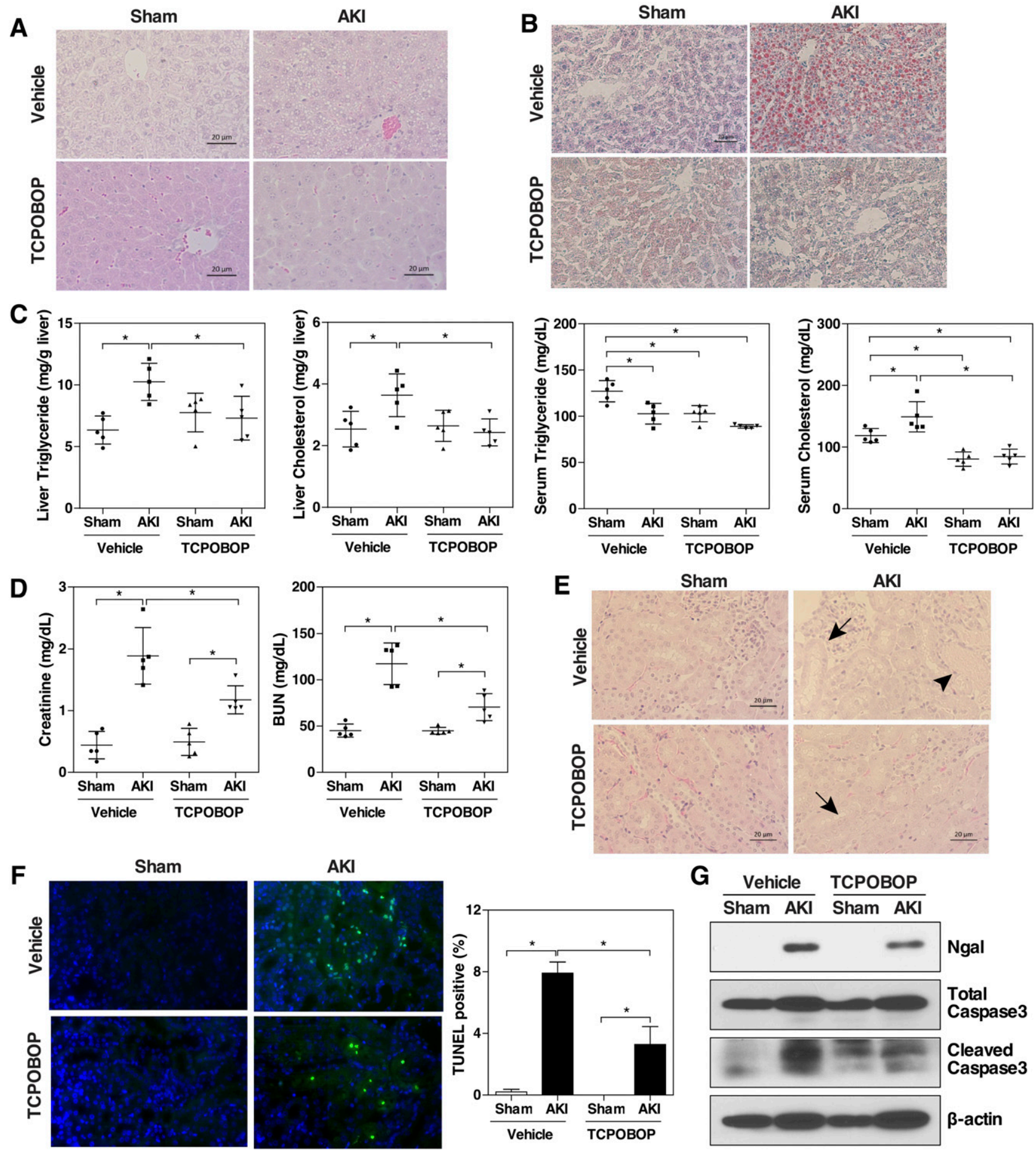

E
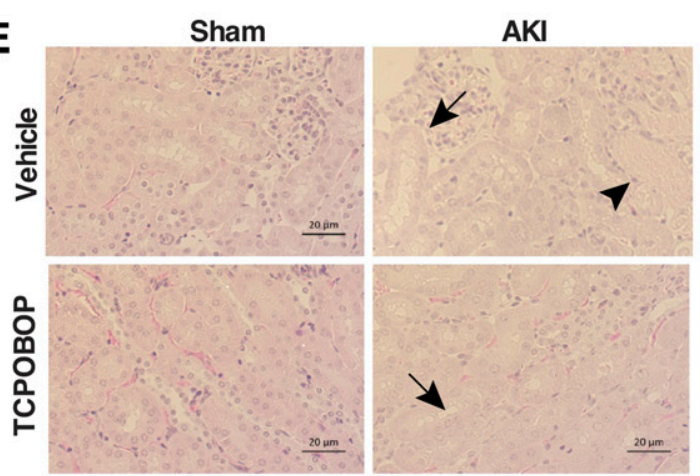

G

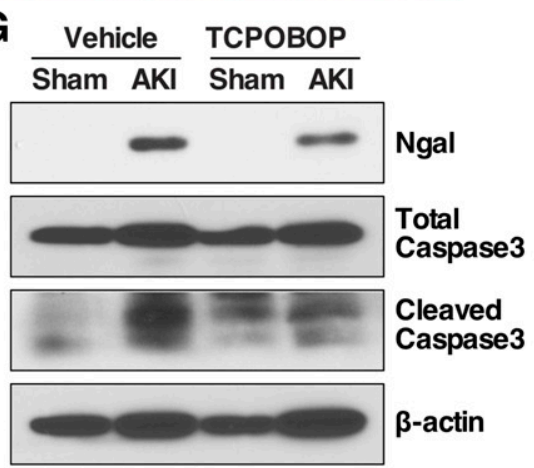

Fig. 6. Activation of CAR attenuates renal IR-induced kidney injury. WT C57BL/6 male mice were randomly assigned, and treatment groups were administered with TCPOBOP for 2 days $(1 \mathrm{mg} / \mathrm{kg}$ body weight per day; i.p.). Vehicle groups received the same volume of corn oil. Mice received the renal IR surgery or sham surgery 24 hours after the last dose of drugs and were sacrificed 48 hours after the renal IR. (A and B) Liver histology as shown by H\&E (A) and Oil-Red O staining (B). (C) Hepatic and serum lipid levels. (D) Serum creatinine and BUN levels. (E) Renal histology as shown by H\&E staining. Arrows and arrowheads indicate tubular dilation and necrosis, respectively. (F) TUNEL staining to identify apoptotic renal cells (green fluorescence). DAPI (4',6-diamidino-2-phenylindole) staining of cell nuclei is shown in blue. Shown on the right is the quantification of the TUNELpositive cells from three mice in each group. (G) Western blot analysis to detect the protein expression of neutrophil gelatinase-associated lipocalin (Ngal), total Caspase3, and cleaved Caspase3 in the mouse kidneys. Each bar represents the mean \pm S.D. of five mice. $* P<0.05$, with the comparisons labeled.

Renal IR Responsive Increase of Serum IL-6 Is a Potential Mechanism for the Renal IR-Induced Fatty Liver and Liver Injury. Inflammatory cytokines and chemokines have been proposed to be the potential mediators of distant organ injury after AKI. It has been suggested that IL-6 may contribute to lung and liver injury after renal ischemia or bilateral nephrectomy in mice (Klein et al., 2008; Park et al., 2011). We then hypothesized that elevation of tissue and 

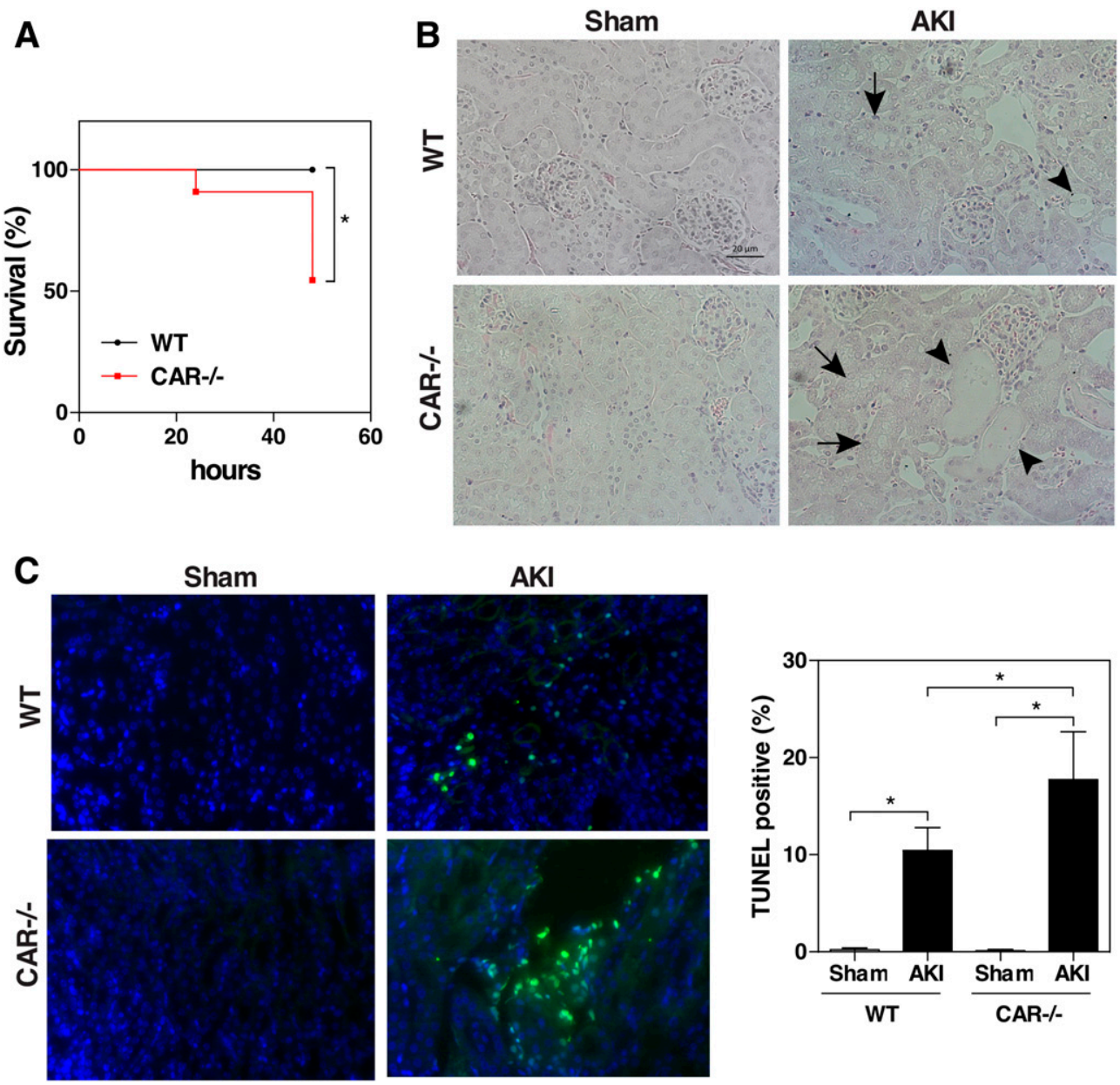

serum levels of IL- 6 may have mediated the renal IR-induced fatty liver and liver injury. Indeed, renal IR increased the mRNA expression of IL- 6 in both the kidney and liver (Fig. $8 \mathrm{~A}$ ), as well as the serum level of IL-6 (Fig. $8 \mathrm{~B}$ ) in the 30-minute ischemia/48-hour reperfusion model. Consistent with the renal and hepatoprotective effect of TCPOBOP, the TCPOBOP-treated AKI mice showed decreased renal and hepatic mRNA expression of IL-6 (Fig. 8A) and serum level of IL-6 (Fig. 8B). In the 30-minute ischemia/24-hour reperfusion model, the serum level of IL- 6 in the $\mathrm{CAR}^{-/-}$mice was also increased, but the level was comparable to that in the WT mice (Fig. 8C), likely because the IL-6 response was maximized. We also showed that treatment of primary hepatocytes with IL-6 suppressed the expression of CAR (Fig. 8D), consistent with a previous report (Pascussi et al., 2000), and this inhibitory effect of IL- 6 on the expression of CAR was abolished upon the TCPOBOP treatment (Fig. 8D). Treatment with IL-6 also inhibited the constitutive and TCPOBOP responsive activity of CAR in a transient transfection and luciferase reporter gene assay (Fig. 8E). As summarized in Fig. 8F, our data suggest that IL-6 may have mediated the renal IR-induced fatty liver and liver injury through the downregulation of the expression and activity of CAR.

\section{Discussion}

Our results showed that renal IR-induced AKI has a distant effect in causing hepatic steatosis and liver injury. The hepatic lipid accumulation may have resulted from decreased expression and activity of CAR and inhibition of VLDL-TG secretion. Pharmacological activation of CAR by TCPOBOP prevented the development of renal IR-induced fatty liver. In contrast, $\mathrm{CAR}^{-/-}$mice showed fatty liver after renal IR, which could not be relieved by TCPOBOP. Alleviation of fatty liver by TCPOBOP also improved kidney function in AKI, suggesting that fatty liver can be considered as a risk factor for kidney injury. We also identified IL-6 as an important mediator for AKI-responsive inhibition of CAR and development of fatty liver.

To our knowledge, this is the first demonstration that renal IR induces CAR downregulation and steatosis in the liver. Although several studies have reported hepatic cellular vacuolization upon renal IR (Golab et al., 2009; Pelletier et al., 2013; Rabadi et al., 2016), understanding the mechanism of renal IR-induced fatty liver has been limited. Most published studies emphasize inflammatory responses, apoptosis, and necrosis in the liver after renal injury. For example, renal peptidyl arginine deiminase- 4 increases the renal tubular inflammatory response after renal IR by interacting with nuclear factor $\kappa \mathrm{B}$ (Rabadi et al., 2016). Genetic ablation of peptidyl arginine deiminase- 4 attenuates AKI-induced kidney injury and liver injury. In this study, we showed that hepatic steatosis is an acute response to renal IR. Renal IR-induced CAR downregulation in the liver resulted in a decreased expression of Mttp, leading to reduced VLDL-TG 

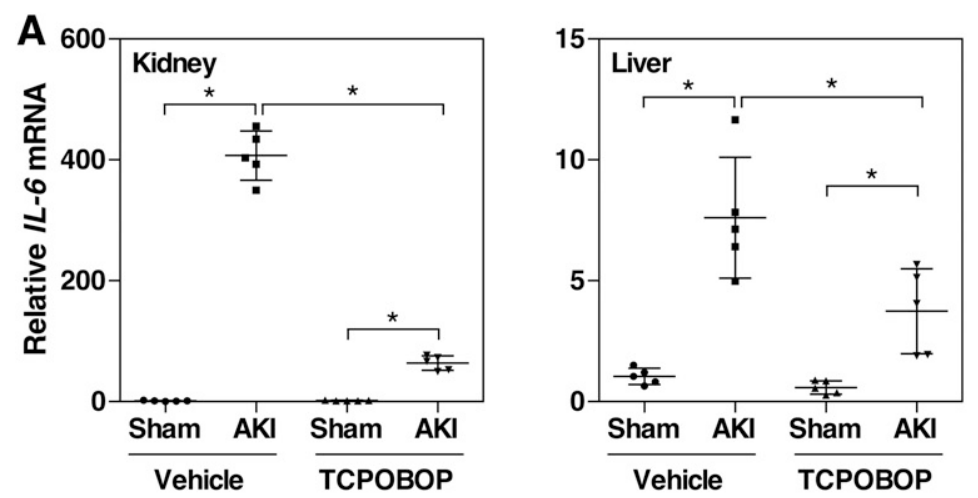

D
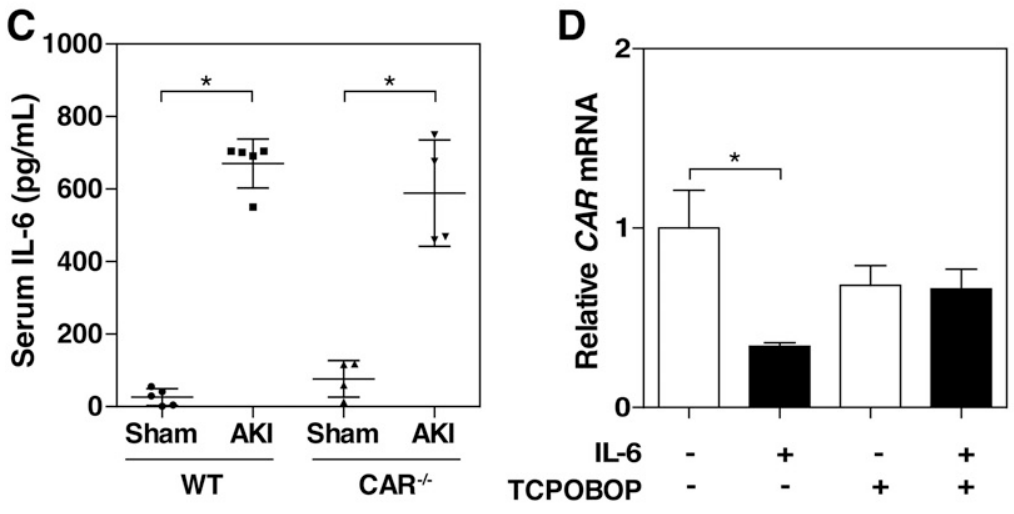

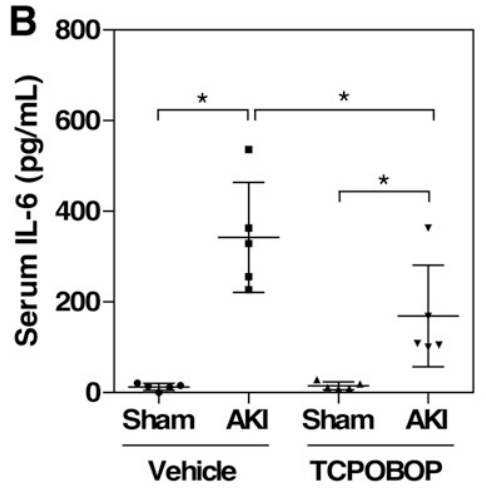

E

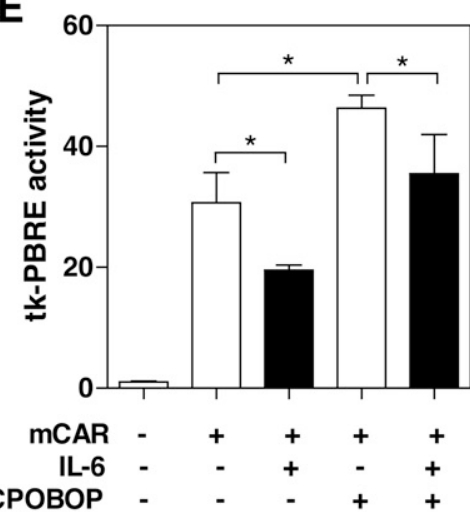

$\mathbf{F}$

CAR

MTTP expression
VLDL-TG secretion

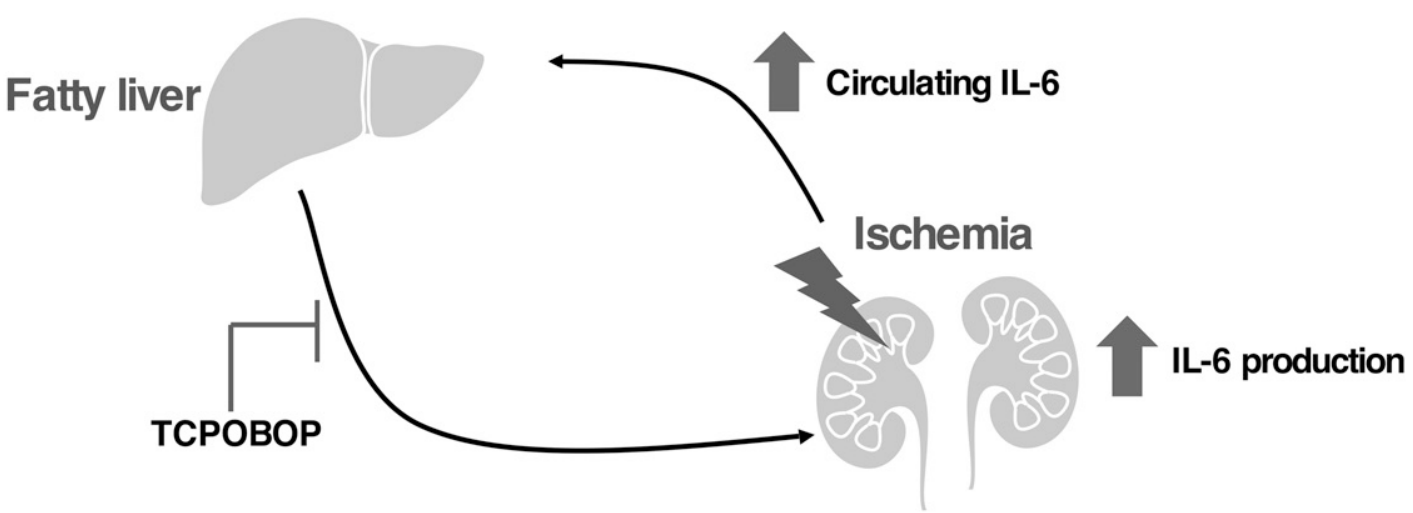

Fig. 8. Renal IR responsive increase of serum IL-6 is a potential mechanism for the renal IR-induced fatty liver and liver injury. (A and B) Mice were subjected to the 30-minute ischemia/48-hour reperfusion model. The $I L-6 \mathrm{mRNA}$ expression in the kidney and liver was determined by quantitative realtime PCR (A), and the serum IL-6 levels were measured by enzyme-linked immunosorbent assay (B). Each bar represents the mean \pm S.D. of five mice. (C) Serum IL-6 levels were measured in WT and $\mathrm{CAR}^{-1-}$ mice that were subjected to the 30 -minute ischemia/24-hour reperfusion model. (D) Mouse primary hepatocytes were isolated and incubated with IL-6 (50 U/ml) in the presence or absence of TCPOBOP $(200 \mathrm{nM})$ for 24 hours. The mRNA expression of CAR was measured by quantitative real-time PCR. (E) HEK293T cells were transiently transfected with the mouse CAR expression vector pCMX-HA-mCAR and the CAR responsive tk-PBRE reporter plasmid. Transfected cells were incubated with IL-6 (50 U/ml) in the presence or absence of TCPOBOP $(200 \mathrm{nM})$ for 24 hours before cell harvesting and luciferase assay. $* P<0.05$, with the comparisons labeled. (F) Proposed mechanisms of renal IR-induced fatty liver and liver injury. Elevation of serum IL-6 after AKI mediates renal IR-induced kidney and liver injury through CAR downregulation.

secretion. Treatment with TCPOBOP abolished the inhibitory effect of renal IR on VLDL-TG secretion and thus decreased lipid accumulation. The mechanism by which CAR blocks the inhibitory effect of renal IR on VLDL-TG secretion remains to be fully understood. Nevertheless, the attenuation of renal IR-induced fatty liver by TCPOBOP is consistent with the antisteatotic activity of CAR (Dong et al., 2009; Gao et al., 2009).

Our findings that renal IR-induced fatty liver and attenuation of fatty liver helped preserve kidney function have clinical implications. Renal transplant recipients have been reported to have a higher prevalence of fatty liver compared 
with the general population (Mikolasevic et al., 2014). Moreover, fatty liver in renal transplant patients was found to have a negative impact on the function of the grafted kidneys. The severity of fatty liver correlated with elevated serum creatinine levels and decreased estimated glomerular filtration rate in renal transplant patients (Anuras et al., 1977; Mikolasevic et al., 2014). In this study, $\mathrm{CAR}^{-/-}$mice were found to be more sensitive to renal IR injury and renal IR-induced lethality, which was likely due to a higher basal triglyceride content in the liver. The spontaneous steatosis in the $\mathrm{CAR}^{-/-}$mice may have resulted from the desuppression of the lipogenic nuclear receptor liver $\mathrm{X}$ receptor (Zhai et al., 2010). In contrast, pharmacological activation of CAR alleviated fatty liver and improved the renal function in AKI. Our results support the notion that fatty liver might be an important risk factor contributing to the development of renal graft dysfunction. Meanwhile, CAR can be explored as a therapeutic target to manage fatty liver and to avoid graft dysfunction in renal transplant patients. Since the expression of CAR in the kidney was negligible, we reason the nephroprotective effect of TCPOBOP was likely due to the hepatic activation of CAR.

Mechanistically, our results suggested the renal IRinduced inflammatory cytokines may have mediated the distal effect of AKI on the liver. Among the inflammatory cytokines, IL-6 plays an important role in AKI-induced distant organ injury (Klein et al., 2008; Park et al., 2011). Elevated levels of serum IL-6 were observed after ischemic AKI and bilateral nephrectomy (Klein et al., 2008). Increases in serum IL-6 have been reported in hospitalized AKI patients, and the level of serum IL-6 showed a significant association with increased mortality (Simmons et al., 2004). IL-6 is known to inhibit the expression of CYP2 and CYP3 genes (Abdel-Razzak et al., 1993; Muntané-Relat et al., 1995; Pascussi et al., 2000; Morgan et al., 2008), likely by decreasing the expression and/or activity of CAR. The elevated hepatic and systemic level of IL-6 may have accounted for the suppression of CAR in the AKI liver. In contrast, treatment with TCPOBOP decreased the hepatic, renal, and circulating levels of IL-6, which helped the recovery of the expression and activity of CAR in the liver, leading to the relief of hepatic and renal injury. The inhibitory effect of TCPOBOP on IL-6 was consistent with the notion that activation of CAR can attenuate inflammation in several disease models (Shah et al., 2014; Cheng et al., 2017).

Another potential implication of our study is that AKI can change hepatic drug metabolism through the downregulation of CAR, a master regulator of drug metabolism. Because the pharmacokinetics and pharmacodynamics of drugs are altered in critically ill patients, dose adjustment is often necessary. Cytochrome P450-mediated drug metabolism is a critical issue in AKI patients due to an altered efficacy or toxicity of drugs. However, the impact of AKI on hepatic drug metabolism is not well documented. Midazolam exhibits a prolonged action in AKI patients due to decreased CYP3A4/5mediated hepatic metabolism as well as diminished renal excretion (Kirwan et al., 2012). Antipyrine and losartan showed a decrease in metabolism by CYP2B 6 and CYP2C9 in animal models of renal IR, bilateral ureteric ligation, and uranyl nitrate injury (Gurley et al., 1997; Yoshitani et al., 2002). The expression of CYP3A4/5, CYP2B6, and CYP2C9 is under transcriptional control by CAR and its sister xenobiotic receptor pregnane X receptor (Xie et al., 2000; Gerbal-Chaloin et al., 2002). Because CAR can also regulate the expression of phase II conjugating enzymes and drug transporters, all stages of hepatic drug metabolism can potentially be affected by AKI due to the suppression of CAR (Xie et al., 2000; Maglich et al., 2002).

In summary, we have uncovered a novel function of CAR in mediating the cross-talk between the injured kidney and liver. Activation of CAR relieved renal IR-induced fatty liver and kidney injury, whereas CAR ablation sensitized mice to renal IR-induced AKI. Knowing that kidney transplant recipients have a higher incidence of fatty liver, and fatty liver has a negative effect on the function of grafted kidneys, it is tempting to speculate that CAR can be explored as a therapeutic target to manage fatty liver and to avoid graft dysfunction in renal transplant patients.

\section{Authorship Contributions}

Participated in research design: Choi, Xie.

Conducted experiments: Choi, Zhou, Barbosa, Niu, Guan, Xu, Ren. Performed data analysis: Choi, Xie.

Wrote or contributed to writing of the manuscript: Choi, Guan, Nolin, Liu, Xie.

\section{References}

Abdel-Razzak Z, Loyer P, Fautrel A, Gautier JC, Corcos L, Turlin B, Beaune P, and Guillouzo A (1993) Cytokines down-regulate expression of major cytochrome $\mathrm{P}-450$ enzymes in adult human hepatocytes in primary culture. Mol Pharmacol 44: 707-715.

Anuras S, Piros J, Bonney WW, Forker EL, Colville DS, and Corry RJ (1977) Liver disease in renal transplant recipients. Arch Intern Med 137:42-48.

Assenat E, Gerbal-Chaloin S, Larrey D, Saric J, Fabre JM, Maurel P, Vilarem MJ, and Pascussi JM (2004) Interleukin 1beta inhibits CAR-induced expression of hepatic genes involved in drug and bilirubin clearance. Hepatology 40:951-960.

Axelsson J, Aström G, Sjölin E, Qureshi AR, Lorente-Cebrián S, Stenvinkel P, and Rydén M (2011) Uraemic sera stimulate lipolysis in human adipocytes: role of perilipin. Nephrol Dial Transplant 26:2485-2491.

Cheng S, Zou M, Liu Q, Kuang J, Shen J, Pu S, Chen L, Li H, Wu T, Li R, et al. (2017) Activation of constitutive androstane receptor prevents cholesterol gallstone formation. Am J Pathol 187:808-818.

Chertow GM, Christiansen CL, Cleary PD, Munro C, and Lazarus JM (1995) Prognostic stratification in critically ill patients with acute renal failure requiring dialysis. Arch Intern Med 155:1505-1511.

Choi HS, Chung M, Tzameli I, Simha D, Lee YK, Seol W, and Moore DD (1997) Differential transactivation by two isoforms of the orphan nuclear hormone receptor CAR. J Biol Chem 272:23565-23571.

Davidson NO and Shelness GS (2000) APOLIPOPROTEIN B: mRNA editing, lipoprotein assembly, and presecretory degradation. Annu Rev Nutr 20:169-193.

Devarajan P (2006) Update on mechanisms of ischemic acute kidney injury. J Am Soc Nephrol 17:1503-1520.

Doi K and Rabb H (2016) Impact of acute kidney injury on distant organ function: recent findings and potential therapeutic targets. Kidney Int 89:555-564.

Dong B, Saha PK, Huang W, Chen W, Abu-Elheiga LA, Wakil SJ, Stevens RD, Ilkayeva O, Newgard CB, Chan L, et al. (2009) Activation of nuclear receptor CAR ameliorates diabetes and fatty liver disease. Proc Natl Acad Sci USA 106: $18831-18836$.

Gao J, He J, Zhai Y, Wada T, and Xie W (2009) The constitutive androstane receptor is an anti-obesity nuclear receptor that improves insulin sensitivity. $J$ Biol Chem 284:25984-25992.

Gao J and Xie W (2012) Targeting xenobiotic receptors PXR and CAR for metabolic diseases. Trends Pharmacol Sci 33:552-558.

Gerbal-Chaloin S, Daujat M, Pascussi JM, Pichard-Garcia L, Vilarem MJ, and Maurel P (2002) Transcriptional regulation of CYP2C9 gene. Role of glucocorticoid receptor and constitutive androstane receptor. J Biol Chem 277:209-217.

Glicklich D, Thung SN, Kapoian T, Tellis V, and Reinus JF (1999) Comparison of clinical features and liver histology in hepatitis C-positive dialysis patients and renal transplant recipients. Am J Gastroenterol 94:159-163.

Golab F, Kadkhodaee M, Zahmatkesh M, Hedayati M, Arab H, Schuster R, Zahedi K, Lentsch AB, and Soleimani M (2009) Ischemic and non-ischemic acute kidney injury cause hepatic damage. Kidney Int 75:783-792.

Gurley BJ, Barone GW, Yamashita K, Polston S, Estes M, and Harden A (1997) Extrahepatic ischemia-reperfusion injury reduces hepatic oxidative drug metabolism as determined by serial antipyrine clearance. Pharm Res 14:67-72.

Honkakoski P, Zelko I, Sueyoshi T, and Negishi M (1998) The nuclear orphan receptor CAR-retinoid $\mathrm{X}$ receptor heterodimer activates the phenobarbitalresponsive enhancer module of the CYP2B gene. Mol Cell Biol 18:5652-5658.

Khwaja A (2012) KDIGO clinical practice guidelines for acute kidney injury. Nephron Clin Pract 120:c179-c184.

Kirwan CJ, MacPhee IA, Lee T, Holt DW, and Philips BJ (2012) Acute kidney injury reduces the hepatic metabolism of midazolam in critically ill patients. Intensive Care Med 38:76-84. 
Klein CL, Hoke TS, Fang WF, Altmann CJ, Douglas IS, and Faubel S (2008) Interleukin-6 mediates lung injury following ischemic acute kidney injury or bilateral nephrectomy. Kidney Int 74:901-909.

Maglich JM, Stoltz CM, Goodwin B, Hawkins-Brown D, Moore JT, and Kliewer SA (2002) Nuclear pregnane $\mathrm{x}$ receptor and constitutive androstane receptor regulate overlapping but distinct sets of genes involved in xenobiotic detoxification. $\mathrm{Mol}$ Pharmacol 62:638-646.

Mikolasevic I, Racki S, Lukenda V, Milic S, Pavletic-Persic M, and Orlic L (2014) Nonalcoholic Fatty liver disease in renal transplant recipients proven by transient elastography. Transplant Proc 46:1347-1352.

Minehira K, Young SG, Villanueva CJ, Yetukuri L, Oresic M, Hellerstein MK, Farese RV, Jr, Horton JD, Preitner F, Thorens B, et al. (2008) Blocking VLDL secretion causes hepatic steatosis but does not affect peripheral lipid stores or insulin sensitivity in mice. J Lipid Res 49:2038-2044.

Morgan ET, Goralski KB, Piquette-Miller M, Renton KW, Robertson GR, Chaluvadi MR, Charles KA, Clarke SJ, Kacevska M, Liddle C, et al. (2008) Regulation of drugmetabolizing enzymes and transporters in infection, inflammation, and cancer. Drug Metab Dispos 36:205-216.

Muntané-Relat J, Ourlin JC, Domergue J, and Maurel P (1995) Differential effects of cytokines on the inducible expression of CYP1A1, CYP1A2, and CYP3A4 in human hepatocytes in primary culture. Hepatology 22:1143-1153.

Musso G, Gambino R, Tabibian JH, Ekstedt M, Kechagias S, Hamaguchi M, Hultcrantz R, Hagström H, Yoon SK, Charatcharoenwitthaya P, et al. (2014) Association of non-alcoholic fatty liver disease with chronic kidney disease: a systematic review and meta-analysis. PLoS Med 11:e1001680.

Park SW, Chen SW, Kim M, Brown KM, Kolls JK, D'Agati VD, and Lee HT (2011) Cytokines induce small intestine and liver injury after renal ischemia or nephrectomy. Lab Invest 91:63-84.

Park SW, Kim M, Kim JY, Ham A, Brown KM, Mori-Akiyama Y, Ouellette AJ, D'Agati VD, and Lee HT (2012) Paneth cell-mediated multiorgan dysfunction after acute kidney injury. J Immunol 189:5421-5433.

Pascussi JM, Gerbal-Chaloin S, Pichard-Garcia L, Daujat M, Fabre JM, Maurel P, and Vilarem MJ (2000) Interleukin-6 negatively regulates the expression of pregnane $\mathrm{X}$ receptor and constitutively activated receptor in primary human hepatocytes. Biochem Biophys Res Commun 274:707-713.

Pelletier CC, Koppe L, Croze ML, Kalbacher E, Vella RE, Guebre-Egziabher F, Géloën A, Badet L, Fouque D, and Soulage CO (2013) White adipose tissue overproduces the lipid-mobilizing factor zinc $\alpha 2$-glycoprotein in chronic kidney disease. Kidney Int 83:878-886.

Rabadi M, Kim M, D'Agati V, and Lee HT (2016) Peptidyl arginine deiminase-4deficient mice are protected against kidney and liver injury after renal ischemia and reperfusion. Am J Physiol Renal Physiol 311:F437-F449.

Rewa O and Bagshaw SM (2014) Acute kidney injury-epidemiology, outcomes and economics. Nat Rev Nephrol 10:193-207.
Shah P, Guo T, Moore DD, and Ghose R (2014) Role of constitutive androstane receptor in Toll-like receptor-mediated regulation of gene expression of hepatic drug-metabolizing enzymes and transporters. Drug Metab Dispos 42 172-181.

Shang Y, Siow YL, Isaak CK, and O K (2016) Downregulation of glutathione biosynthesis contributes to oxidative stress and liver dysfunction in acute kidney injury. Oxid Med Cell Longev 2016:9707292.

Shemin D and Dworkin LD (2011) Neutrophil gelatinase-associated lipocalin (NGAL) as a biomarker for early acute kidney injury. Crit Care Clin 27:379-389.

Shi Y, Melnikov VY, Schrier RW, and Edelstein CL (2000) Downregulation of the calpain inhibitor protein calpastatin by caspases during renal ischemia-reperfusion. Am J Physiol Renal Physiol 279:F509-F517.

Simmons EM, Himmelfarb J, Sezer MT, Chertow GM, Mehta RL, Paganini EP, Soroko S, Freedman S, Becker K, Spratt D, et al.; PICARD Study Group (2004) Plasma cytokine levels predict mortality in patients with acute renal failure. Kidney Int 65:1357-1365.

Targher G and Byrne CD (2017) Non-alcoholic fatty liver disease: an emerging driving force in chronic kidney disease. Nat Rev Nephrol 13:297-310.

Venkatachalam MA, Weinberg JM, Kriz W, and Bidani AK (2015) Failed tubule recovery, AKI-CKD transition, and kidney disease progression. J Am Soc Nephrol 26:1765-1776.

Wei P, Zhang J, Egan-Hafley M, Liang S, and Moore DD (2000) The nuclear receptor CAR mediates specific xenobiotic induction of drug metabolism. Nature 407 920-923.

Wetterau JR, Lin MC, and Jamil H (1997) Microsomal triglyceride transfer protein Biochim Biophys Acta 1345:136-150.

Wu H, Chen G, Wyburn KR, Yin J, Bertolino P, Eris JM, Alexander SI, Sharland AF, and Chadban SJ (2007) TLR4 activation mediates kidney ischemia/reperfusion injury. J Clin Invest 117:2847-2859.

Xie W, Barwick JL, Simon CM, Pierce AM, Safe S, Blumberg B, Guzelian PS, and Evans RM (2000) Reciprocal activation of xenobiotic response genes by nuclear receptors SXR/PXR and CAR. Genes Dev 14:3014-3023.

Yoshitani T, Yagi H, Inotsume N, and Yasuhara M (2002) Effect of experimenta renal failure on the pharmacokinetics of losartan in rats. Biol Pharm Bull 25: 1077-1083.

Zhai Y, Wada T, Zhang B, Khadem S, Ren S, Kuruba R, Li S, and Xie W (2010) A functional cross-talk between liver $\mathrm{X}$ receptor- $\alpha$ and constitutive androstane receptor links lipogenesis and xenobiotic responses. Mol Pharmacol 78:666-674.

Address correspondence to: Center for Pharmacogenetics and Department of Pharmaceutical Sciences, 306 Salk Pavilion, University of Pittsburgh, Pittsburgh, PA 15261. E-mail: wex6@pitt.edu 